\title{
TRAFFIC-SIGN RECOGNITION FOR AN INTELLIGENT VEHICLE/DRIVER ASSISTANT SYSTEM USING HOG
}

\author{
Karthiga.PL ${ }^{1}$ S.Md.Mansoor Roomi ${ }^{2}$, Kowsalya.J ${ }^{3}$ \\ ${ }^{1}$ PG Student, Department of Electronics and Communication Engineering, TCE, Madurai \\ ${ }^{2}$ Assistant Professor, Department of Electronics and Communication Engineering, TCE, \\ Madurai \\ ${ }^{3}$ UG Student, Department of Electronics and Communication Engineering, TCE, \\ Madurai
}

\begin{abstract}
In order to be deployed in driving environments, Intelligent transport system (ITS) must be able to recognize and respond to exceptional road conditions such as traffic signs, highway work zones and imminent road works automatically. Recognition of traffic sign is playing a vital role in the intelligent transport system, it enhances traffic safety by providing drivers with safety and precaution information about road hazards. To recognize the traffic sign, the system has been proposed with three phases. They are Traffic board Detection, Feature extraction and Recognition. The detection phase consists of RGBbased colour thresholding and shape analysis, which offers robustness to differences in lighting situations. A Histogram of Oriented Gradients (HOG) technique was adopted to extract the features from the segmented output. Finally, traffic signs recognition is done by $k$-Nearest Neighbors $(k-N N)$ classifiers. It achieves an classification accuracy upto $63 \%$.
\end{abstract}

\section{KEYWORDS}

Intelligent Transport System, Road Sign Recognition, RGB-based colour thresholding, HOG, $k$-NN Classifier

\section{INTRODUCTION}

Traffic-sign recognition (TSR) is an fundamental component of a driver assistance system (DAS)[6]. It enhances safety by notifying the driver of speed limits or possible dangers such as imminent road works or pedestrian crossings. The traffic signs have two utmost properties are their 'Colour' and 'Shape' using which the real-time system becomes capable to detect and identify the Traffic Signs e.g., red-rimmed circular prohibition signs, triangular warning signs, and blue information signs. The simplified pictograms make them easily perceivable and understandable.

The detection and recognition of these traffic signs may face one or more of the following difficulties: (i) Faded signs(ii) The presence of obstacles in the scene(iii) Similar objects in the scene or similar background color (iv) Damaged signs. To overcome these difficulties many techniques have been suggested. 


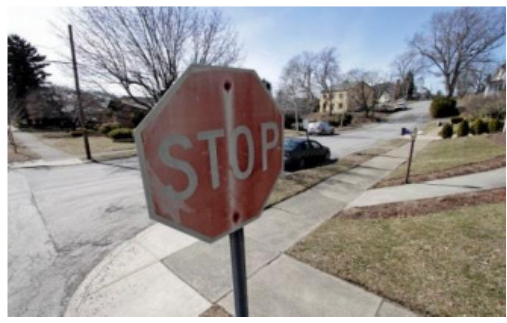

(a)

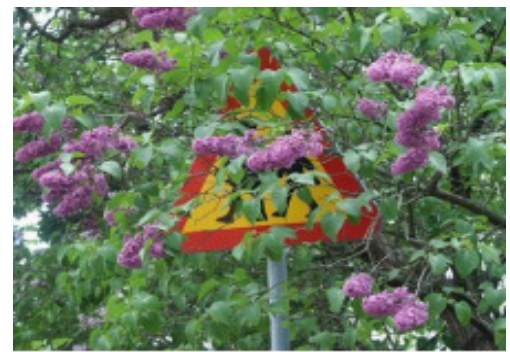

(c)

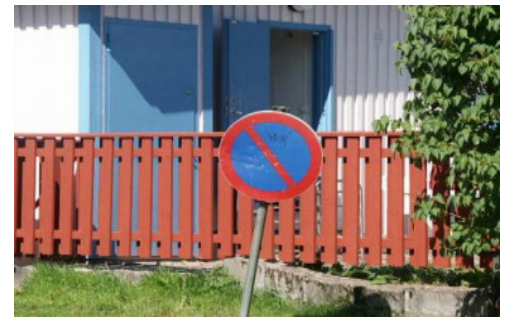

(b)

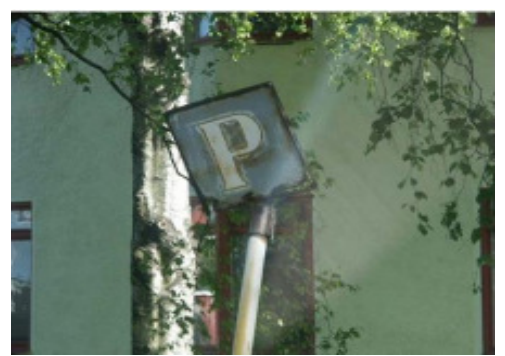

(d)

Fig. 1. Examples for difficulties facing the TSR task: (a) Faded signs, (b) Similar objects in the scene or similar background color, (c) The presence of obstacles in the scene, (d)Damaged signs.

S. Maldonado et al. [1] used HSI color space for detection and then used SVM for classification of traffic signs. L.W.Tsai et ale [2] proposed a different color model for making road sign colors more squeezed. After the transformation, the RBF network is then used to excerpt road sign candidates and then a rectification process is also applied to rectifying each slanted road. X.W.Gao et ale [3] applied the color advent model CIECAM97 to extract color information and extract the shape features by FASTS model to segment and classify the traffic signs. S. Prieto et al. [4] used a kind of ANN called SAM at every level of the detection and recognition of RSs. A. de la Escalera et al. [6] used a inherent algorithm for the detection step, agree to invariance localization, scale, rotation, weather conditions, partial occlusion. Then the classification is done by using neural network classifier.

There are many methods to excerpt features from road signs for classification. P. Paclik et al. [8] use proposed the scale change invariant normalized central moments and compactness from binary images. H. Fleyeh et al. [9] has summarized Zernike moments as features. A fuzzy ARTMAP is trained directly with features or by using KLT for dimension reduction, or by using LDA algorithm as data separation and dimension reduction algorithm

In this paper the goal is to propose a new intelligent transport system to identify the red road signs that could be used for intelligent vehicles. In these vehicles an image of road scene is captured by a camera and then the type of traffic sign is determined. The system has three major steps: Traffic board detection, feature extraction, and classification.

\section{Charectristics of Roadsign}

Traffic signs have been designed so that they are easily distinct from driving environment. The colour for traffic sign are chosen in a way such that, it serves different purposes and is also distinguishable for the driver while driving. The signs are characterized by fixed shapes like triangle, circle, octagon, and rectangle. The combined feature of shape and colour are used by the 
Computer Science \& Engineering: An International Journal (CSEIJ), Vol.6, No.1, February 2016

driver to distinguish a traffic sign. Hence an autonomous system also uses the same principle of the colour and shape of the traffic signs. With respect to the road the traffic signs are positioned at well-defined locations so that the drivers can expect the position of the sign. The road sign may contain text as a string of characters, picture or both to represent the meaning of the sign. They are characterized by using fixed text fonts and character heights. There are a large number of traffic signs in India categorized as WARNING, COMPULSORY, REGULATORY and INFORMATORY. This makes a total of 92 traffic signs all together. These signs are mainly characterized by colour and shape. Figure 2 shows the different types of Indian traffic sign,

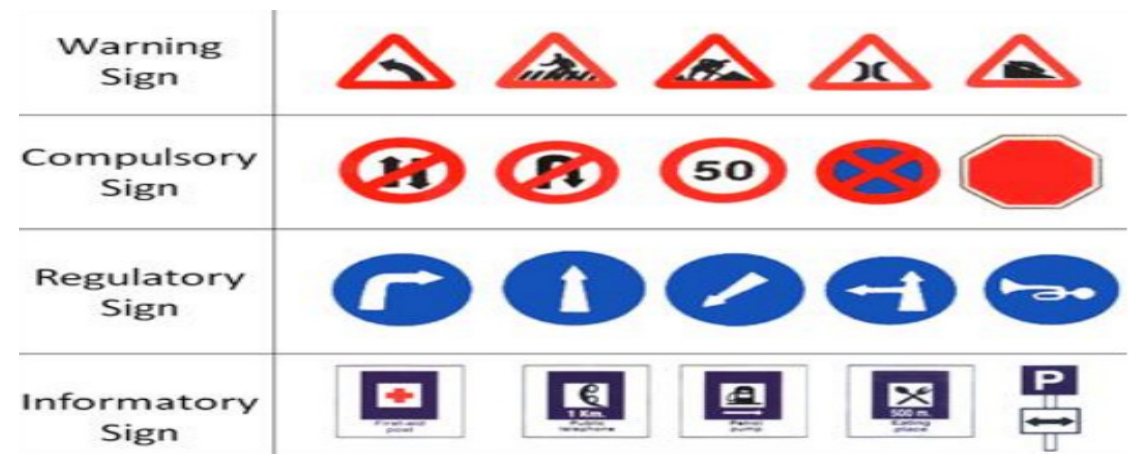

Fig. 2 Different category of traffic signs

\section{PROPOSED APPROACH}

Our proposed approach uses the HOG feature and k-NN classifier to efficiently classify the traffic-sign images. It enhances the classifier performance and improve the performance under various illumination conditions like under or over illumination. Fig. 2 shows the proposed approach.

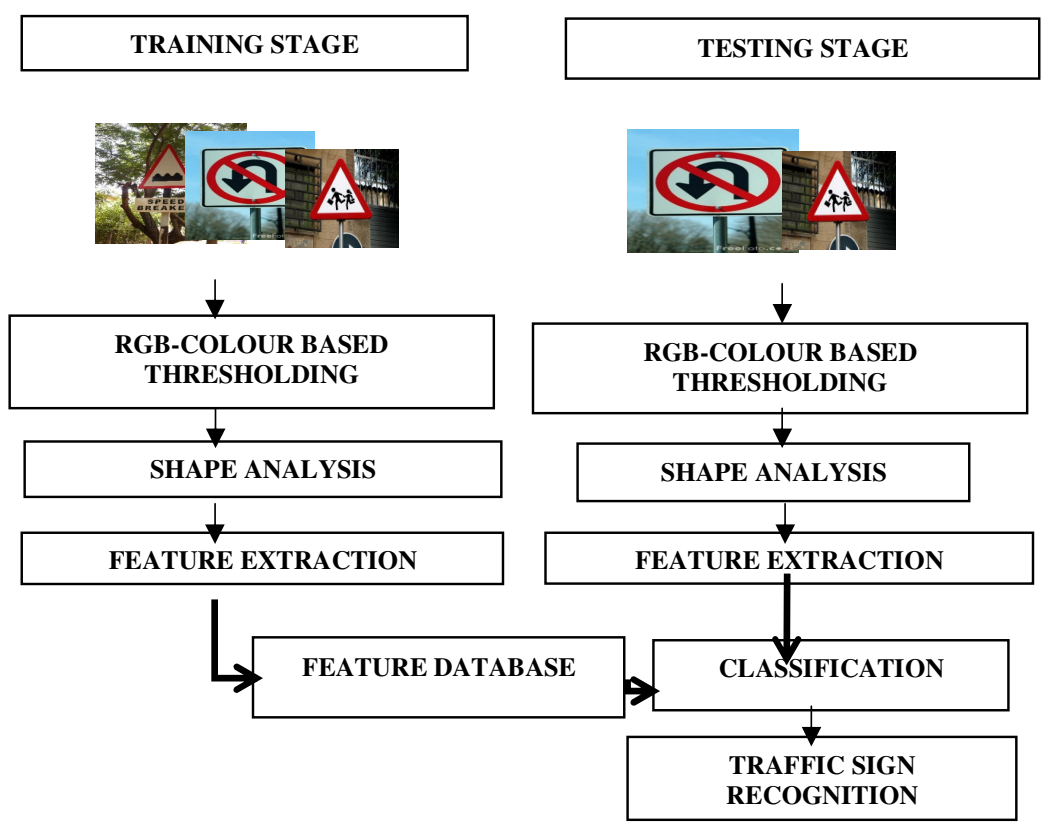

Figure 2: Proposed approach 


\subsection{RGB-BASED COLOUR THRESHOLdiNG}

Colour thresholding is a common technique in traffic sign detection and there are different methods. Since most traffic signs contain red or blue colours, thresholding techniques concentrate mostly on these two colours. One technique is to make use of Normalized RGB (RGBN) colour space, presented in (Gomez-Moreno, Maldonado-Bascon, Gil-Jimenez, \& Lafuente-Arroyo, 2010). This is used to decrease the effects of illumination changes and also allows to find the correct thresholds. The mask equations for red and blue colours are the following

$\operatorname{Re} d(i, j)=\left\{\begin{array}{l}\text { True, if } \mathrm{r}(i, j) \geq g(i, j) \text { and } r(i, j) \geq b(i, j) \text { and } g(i, j) /|r(i, j)-g(i, j)| \leq T h R_{1} \\ \text { False, otherwise }\end{array}\right.$

$\operatorname{Blue}(i, j)=\left\{\begin{array}{l}\text { True, if } \mathrm{b}(i, j) \geq r(i, j) \text { and } g(i, j) / b(i, j) \leq T h B_{1} \\ \text { False, otherwise }\end{array}\right.$

Where $\mathrm{ThR}_{1}$ and $\mathrm{ThB}_{1}$ threshold values for red and blue band, these values are computed by using binary thresholding method.

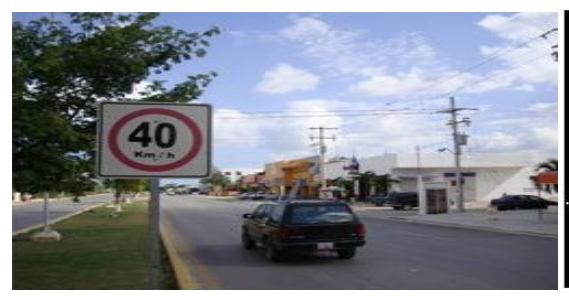

(a)

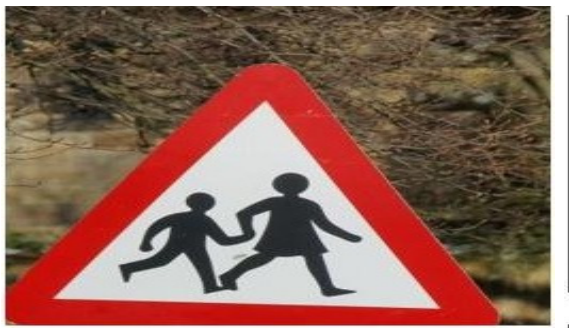

(a1)

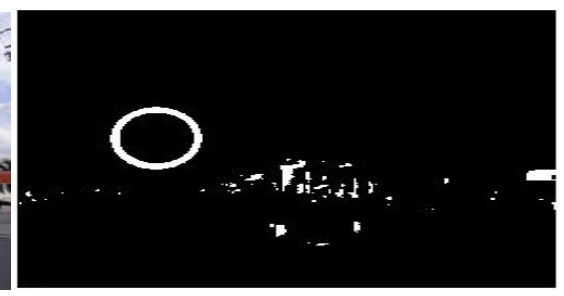

(b)

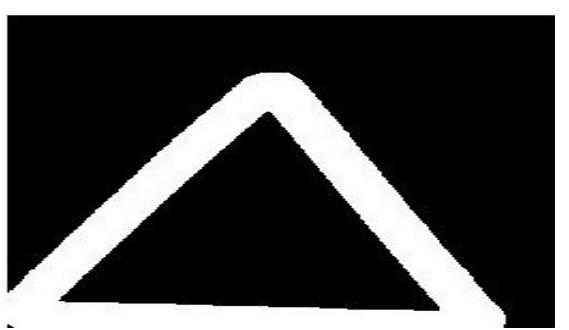

(b1)

Figure:3 (a) Original image (a1) Original image (b) Detected Traffic Sign Board image (b1)Detected Traffic Sign Board image

\subsection{Shape Analysis}

The main task of shape analysis includes cleaning the binary image from noise and Small objects, applying connected components labelling algorithm and identifying the traffic sign. This module recognized road traffic sign so that it becomes invariant to the in-plane transformations. This means that the consequential sign has a fixed size and it is located in a standard position where its center of gravity is located in the center of the image. 


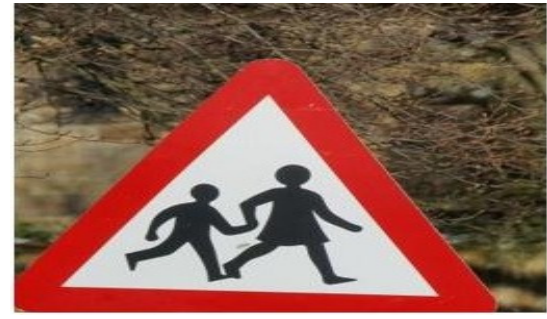

(a)

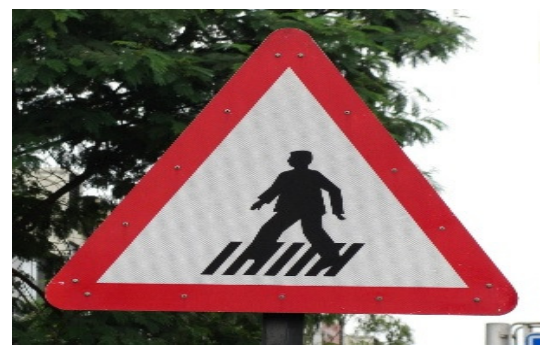

(a1)

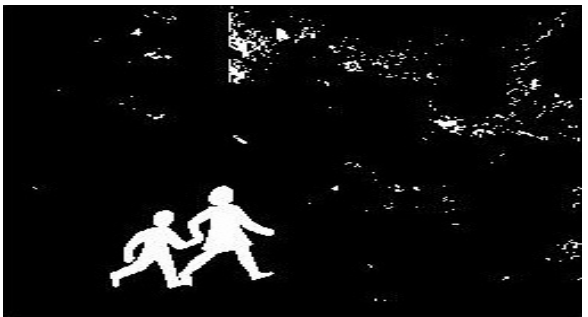

(b)

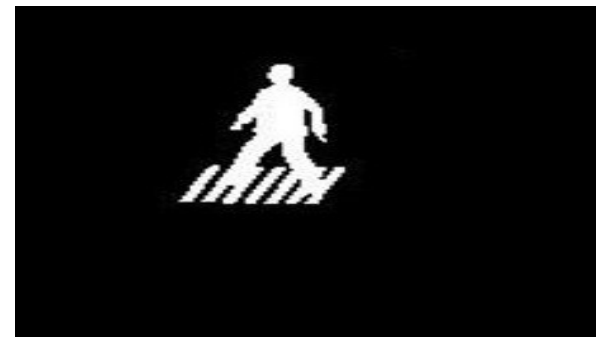

(b1)

Figure 4 (a) original image (a1)original image (b) Results of shape analysis analysis

(b1)Results of shape

\subsection{HOG FEATURE EXTRACTION}

The HOG was proposed by Dalal and Triggs [12] and primarily used for pedestrian detection. Fig. 5 shows the structure of the HOG descriptor. The image is divided into overlapping blocks. Each block, in turn, is divided into non overlapping cells. The gradient orientation and magnitude are computed for each pixel. A histogram of these orientations is formed for each cell. The magnitude of the gradient is used as a vote weight. A local contrast normalization of the blocks improves the pedestrian detection results. The resulting histograms are concatenated to form the HOG descriptor.

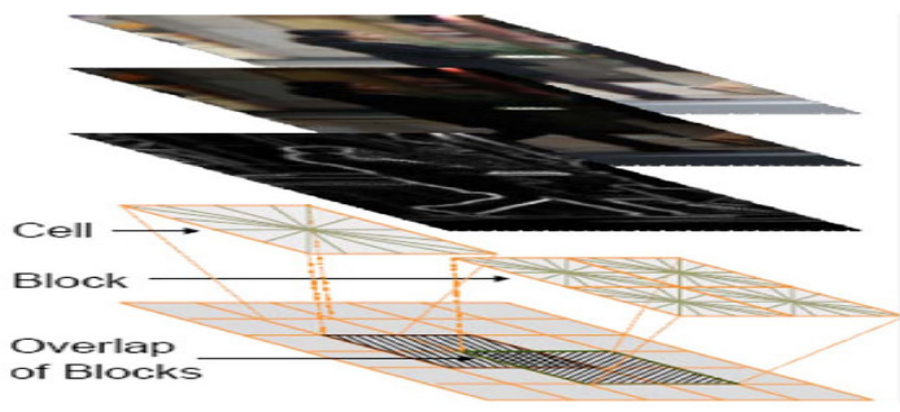

Figure 5: structure of HOG descriptor

The gradient magnitude and orientation is given by,

$$
\begin{aligned}
& \operatorname{mag}(\nabla f)=\sqrt{G_{x^{2}}+G_{y^{2}}} \approx\left|G_{x}\right|+\left|G_{y}\right| \\
& \arg =\tan -1\left(\frac{G_{y}}{G_{x}}\right)
\end{aligned}
$$


Where $G_{x}$ is the image filtered by horizontal mask and $G_{y}$ is the image filtered by vertical mask

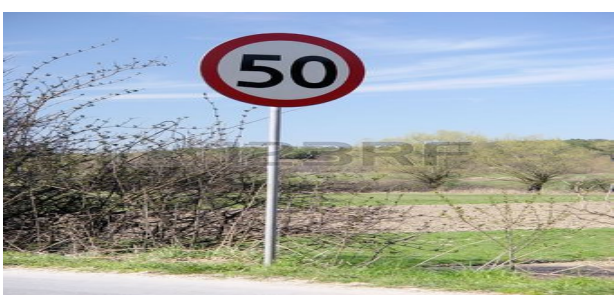

(a)

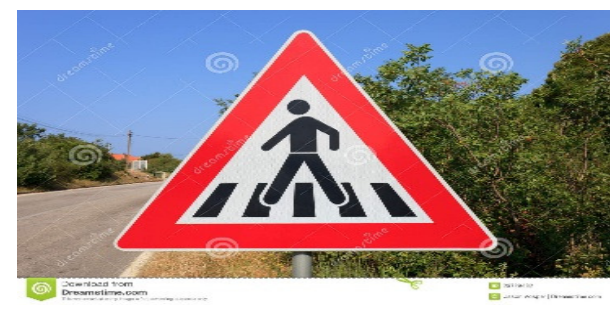

(a1)

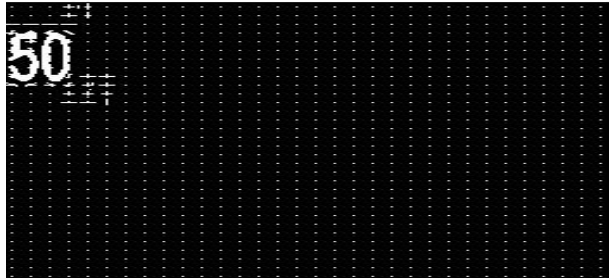

(b)

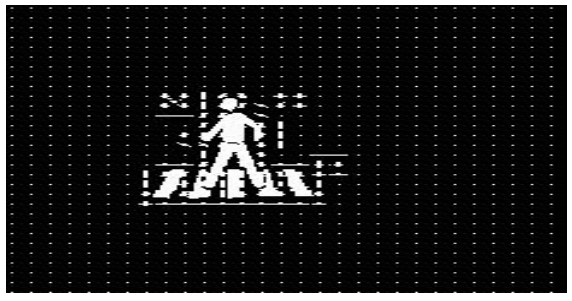

(b1)

Figure6:(a) Original image (a1)Original image(b) HOG feature extracted image (b1)HOG feature extracted image

\subsection{K-NeAReSt Neighbor Classifier}

The idea in k-Nearest Neighbor method is to identify k samples in the training set whose liberated variable $\mathrm{x}$ are similar to $\mathrm{u}$ and we use these $\mathrm{k}$ samples to classify this new sample into a class $\mathrm{v}$. If all we are prepared to assume is that $\mathrm{f}$ is a smooth function, a practical idea is to look for samples in our training data that are near (in terms of the independent variables) and then we have to compute $\mathrm{v}$ from the values of $\mathrm{y}$ for these samples.

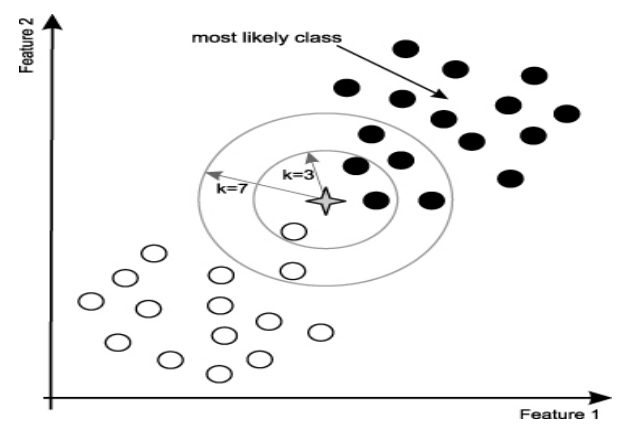

Figure 7:k-NN classifier approach

When it talk about neighbors it suggest that there is a distance or dissimilarity measure that it can compute between samples based on the independent variables. For the moment it would concern ourselves to the most popular measure of distance: Euclidean distance.

The Euclidean distance between the points $\mathrm{x}$ and $\mathrm{u}$ is 


$$
\mathrm{d}(\mathrm{x}, \mathrm{u})=\sqrt{\sum_{i=1}^{n}(x i-y i)^{2}}
$$

It examine other ways to measure distance between points in the space of independent analyst variables when we discuss clustering methods. The simplest case is $\mathrm{k}=1$ where we have to find the sample in the training data which is closest (the nearest neighbor) to $\mathrm{u}$ and set $\mathrm{v}=\mathrm{y}$ where $\mathrm{y}$ is the class of the nearest neighboring sample.In the proposed approach traffic signs are classified by using k-NN classifier.In this approach using 18 different classes like(school zone,one way,two way,speed breaker etc..,) are used.

\section{EXPERIMENTAL RESULTS AND DiSCUSSION}

\subsection{Sample Database Images}

A database comprising of 200 images is created for evaluation of the proposed algorithm. The dataset consists of all variety of traffic signs like warning sign, prohibitory sign, information sign and mandatory sign. The images are captured under varying scale and illumination conditions. . Some of the traffic sign images are shown in figure 8.

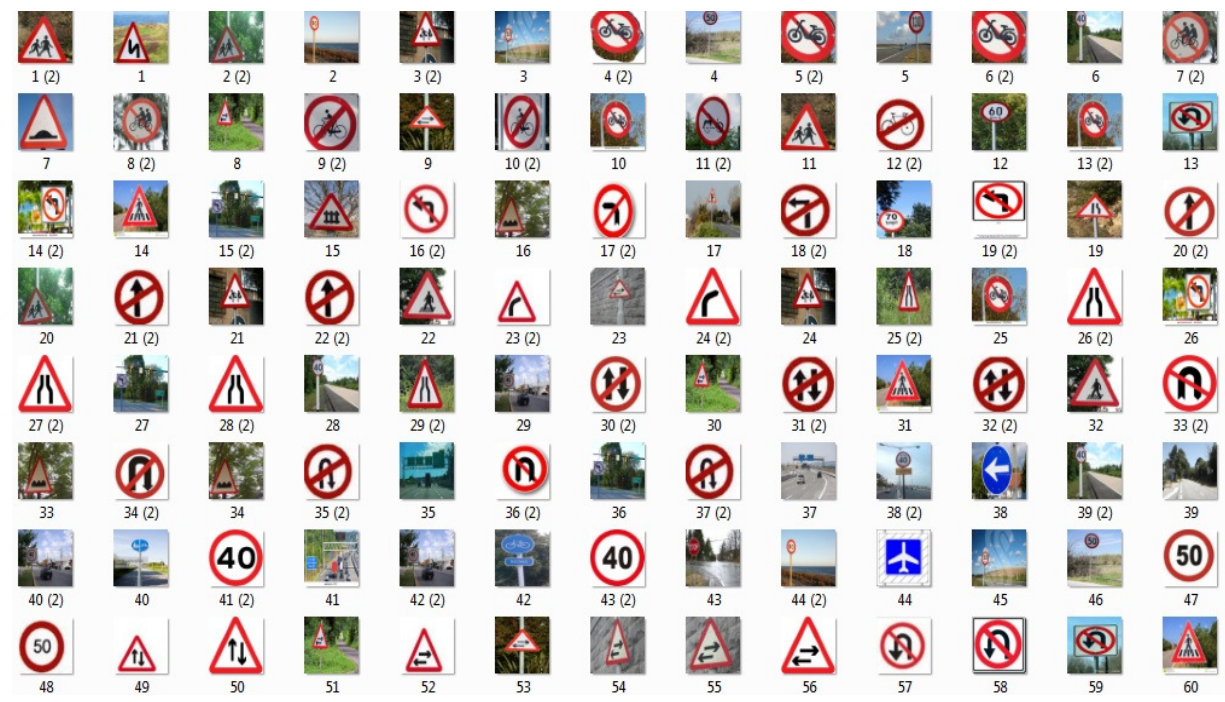

Figure 8: sample database images

\subsection{Experimental Results}

In this section, the exhaustive experimentation of the proposed methodology on the created dataset has been presented. From the experimental analysis it is observed that the proposed methodology works better results for traffic sign recognition. The proposed methodology based on HOG feature is experimented for 18 different classes like school zone,40 km speed limit,50 $\mathrm{km}$ speed limit, pedestrian crossing, speed breaker, two way crossing and one way crossing etc.., Initially, the traffic sign board is detected from the input images using RGB based color segmentation algorithm. After color segmentation, the segmented image undergoes a post processing step using morphological operations. Next, the Histogram of oriented gradients of the segmented image is computed which yields five orientation angles as theta=0, 45, 90, 135 and 
180 degree. Finally the histogram of the edge descriptor is the feature vector of the proposed algorithm. The obtained feature vector is classified using the traditional k-NN classifier. The proposed algorithm for traffic sign recognition attains a classification accuracy of $63 \%$. Graphical User Interface (GUI) results of the proposed algorithm is shown in Figure 9,

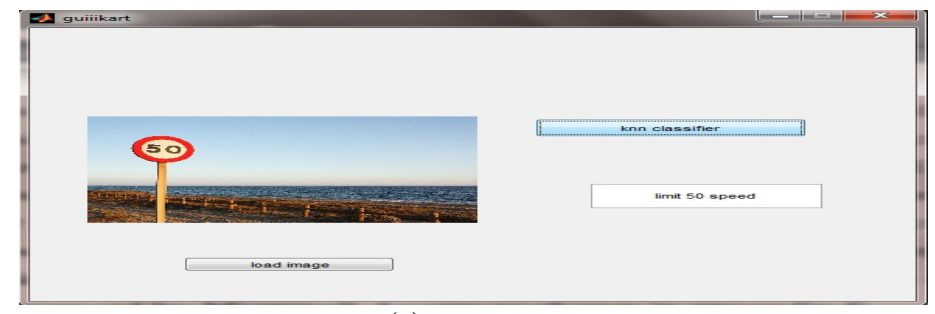

(a)

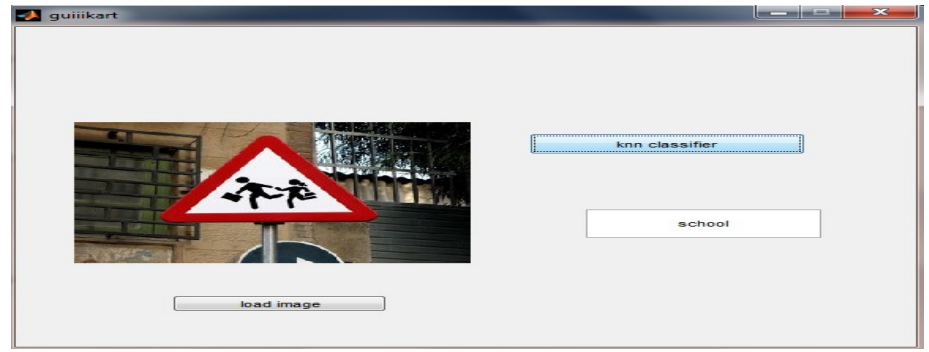

(b)

Figure 9 : (a) Results of $50 \mathrm{~km}$ speed limit sign image (b) Results of school zone image

\section{CONCLUSIONS}

This paper proposes a new and effective method for both detection and recognition of traffic signs. The proposed system uses a very simple and accurate detection procedure and classifies the detected image with a accurate feature extraction algorithm along with a classifier. The proposed strategy comprises three main sections. At first the road sign image must be detected from road scene image. The RGB-based colour thresholding was used for detection. Then for extraction of features using HOG. Finally the feature vector of road sign is classified using k-NN classifier. Experimental results indicate that the proposed system is strong against non-uniform illuminations, scale and rotations. But it has some defects. For instance if there is more than one red road sign in the image, the response will not be unique. So some improvements remain as tasks for the future. Hence the proposed recognition procedure could be used also for recognition of the blue road signs and as the future task we'll improve our system to detect and recognize the road signs in complex background scenario and we will increase the number of road sign classes.

\section{REFERENCES}

[1] S. M . Basc6n , S. L. Arroyo , P. G . Jirnenez.H . G . Moreno,F.L.Ferreras,( June 2007 )"Road-Sign Detection and Recognition Based on Support Vector Machines" , IEEE Transactions on Intelligent Transportation Systems, vol. 8, no. 2,

[2] L. W . Tsai , J . W . Hs ieh, C. H . Chuang, Y . J . Ts en g, K. C. Fan , C . C.Lee , H. Poor(2008 ), "Road sign detection using eigen colour ", lET Comput.Vis .,Vol. 2, No .3, pp. 164-177.

[3] X.W . Gao , L. Podladchikova, D. Shaposhnikov, K. Hon g, N.shevtsova,(2006) ,"Recognition of traffic signs based on their colour and shape features extracted using human vision models", J . Vis .Commun . Image R. $17675-685$. 
Computer Science \& Engineering: An International Journal (CSEIJ), Vol.6, No.1, February 2016

[4] M . S. Prieto , A. R. Allen (2008)," Using self-organising maps in the detection and recognition of road signs ", Image and Vision Computing.

[5] C .Y . Fang , C .S. Fuh , P.S. Yen, S . Cherng , S.W . Chen (2004)," An automatic road sign recognition system based on a computational model of human recognition processing", Computer Vision and Image Understanding $96237-268$.

[6] A. Escalera, J .M . Armingol, M . Mata(2003) ,' Traffic sign recognition and analysis for intelligent vehicles", Image and Vision Computing 21247 -258.

[7] J .Sheeba Rani , D.Devaraj , R . Sukanesh, (2007 )" A Novel Feature Extraction for Face Recognition"International Conference on Computational Intelligence and Multimedia Applications.

[8] P. Paclik , J . Novovicova, P. Pudil , P. Somol, (2000) " Road sign classifcation using Laplace kernel classifer" , Pattern Recognition Letters 21 I 165-1173.

[9] H. Fleyeh, M . Dougherty, D. Aenugula, S. Baddam,( 2007) "Invariant Road Sign Recognition with Fuzzy ARTMAP and Zernike" , Proceedings of the 2007 IEE E Intelligent Vehicles Symposium Istanbul, Turkey, June 13-15, .

[10] W. Y.Wu , T . C. Hsieh , C. S. Lai, "Extracting Road Signs using the Color Information" , International Journal of Computer Science and Engineering Volume 2 Number I .

[11] Gomez-Moreno, H., Maldonado-Bascon, S., Gil-Jimenez, P., \& Lafuente-Arroyo, S.(2010). Goal evaluation of segmentation algorithms for traffic sign recognition. IEEE Transactions on Intelligent Transportation Systems, 11, 917-930.

[12] Navneet Dalal and Bill Triggs, "Histograms of Oriented Gradients for Human Detection", IJCV, 60(2):91-110.

\section{AUTHORS}

PL.Karthiga received her B.E.degree from Alagappa Chettiar College of Engineering and Technology, Karaikudi in 2014 and is pursuing her M.E. Wireless Technology at Thiagarajar college of Engineering, Madurai.

S. Mohamed Mansoor Roomi received his B.E.degree from Madurai Kamaraj University, in 1990, his M.E. degree in Power Systems and Communication Systems from Thiagarajar College of Engineering in 1992 and 1997 and his Ph.D.in Image Analysis from Madurai Kamaraj University in 2009. He has authored and co-authored more than 150 papers in various journals and conference proceedings and numerous technical and industrial project reports.
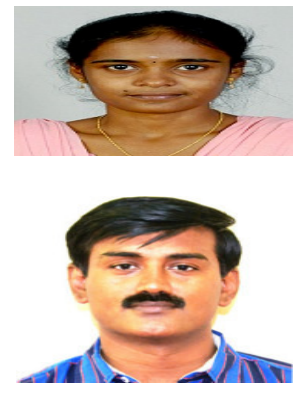

J.Kowsalya pursuing her B.E.Electronics and Communication Engineering at Thiagarajar college of Engineering, Madurai.

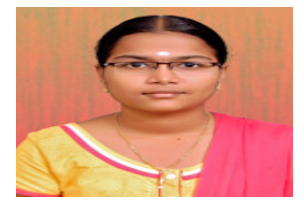

